\title{
A Novel Nanoionics-based Switch for Microwave Applications
}

\author{
James A. Nessel ${ }^{1}$, Richard Q. Lee ${ }^{1}$, Carl H. Mueller ${ }^{2}$, Michael N. Kozicki ${ }^{3}$, Minghan Ren ${ }^{3}$, and Jacki \\ Morse $^{3}$ \\ ${ }^{1}$ NASA Glenn Research Center, Cleveland, OH, 44135, USA \\ ${ }^{2}$ Analex Corporation, Cleveland, $\mathrm{OH}, 44135$, USA \\ ${ }^{3}$ Arizona State University, Tempe, AZ, 85287, USA
}

\begin{abstract}
This paper reports the development and characterization of a novel switching device for use in microwave systems. The device utilizes a switching mechanism based on nanoionics, in which mobile ions within a solid electrolyte undergo an electrochemical process to form and remove a conductive metallic "bridge" to define the change of state. The nanoionics-based switch has demonstrated an insertion loss of $\sim 0.5 \mathrm{~dB}$, isolation of $>30 \mathrm{~dB}$, low voltage operation (1V), low power $(\sim \mu W)$ and low energy $(\sim n J)$ consumption, and excellent linearity up to $6 \mathrm{GHz}$. The switch requires fewer bias operations (due to non-volatile nature) and has a simple planar geometry allowing for novel device structures and easy integration into microwave power distribution circuits.
\end{abstract}

Index Terms - microwave switches, nanotechnology.

\section{INTRODUCTION}

Nanoionics is an emerging field receiving increasing interest in the development of novel nanotech devices [1]-[4]. This technology concerns itself with materials and devices that rely on ion transport and chemical change at the nanoscale. The chemical change can take the form of an oxidation/reduction reaction of ionic metal species within some base material to essentially "grow" metal on the surface (or within) a film at low energies. Exploiting this effect is the basis for devices such as resistance-change memory cells [1][2], tunable resonators [3], and microfluidic valves [4].

In this paper, we report on the first development of a novel switching mechanism for microwave applications based on nanoionic materials. Because the nanoionics-based switch is electrochemical in nature, several unique advantages become apparent which gives this technology the potential to overcome some of the issues in solid state and radiofrequency microelectromechanical (RF MEMS)-based systems. Firstly, the process is non-volatile. No power is required to maintain a particular state (ON or OFF), but only to change states. As a result, fewer bias operations are needed. The process is also low energy, requiring on the order of $\mathrm{nJ}$ to operate. Power consumption is thus comparable to MEMS devices. There are no moving parts (as in MEMS devices), eliminating this point of failure and enhancing speed. To activate a nanoionic device, only on the order of tenths of a volt is necessary, superior to solid state and MEMS-based switches. Metal ion conductivity in these base films is comparable to electron conductivity in semiconductors, indicating the switching speed has the potential to be competitive with solid state switches. Finally, the fabrication process of nanoionics-based switches is trivial in nature, requiring only as little as 5 processing steps using common semiconductor fabrication tools. Compared to solid state and MEMS devices, which may require several sacrificial layers and masks for fabrication, this should result in an unparalleled ease of integration and reduction in cost.

\section{NANOIONIC Device Physics (Overview)}

The fundamental operation of the nanoionic switch is rooted in the phenomenon of ion conduction in solid electrolytes. Synonymous with liquid electrolytes (e.g., lead-acid batteries), solid electrolytes consist of mobile ions which undergo oxidation/reduction reactions at the anode and cathode of the system. The fundamental difference between solid electrolyte and liquid electrolyte behavior is that the mobile ions are of a single polarity while the opposite polarity species remain fixed. The fixed ions essentially create a solid matrix in which mobile ions can "hop" into neighboring potential wells. Based on this short-range hopping mechanism for conduction, the ionic conductivity of solid electrolytes can locally approach electronic conductivity levels realizable in today's semiconductors [1].

Many inorganic and organic materials can conduct ions to some extent but it is the compounds of elements in the column of the Periodic Table headed by oxygen, the so-called chalcogens, that are of most interest in the context of electrochemical switching devices, principally because of their high ion availability and mobility at normal device operating temperatures. When a metal ion is introduced into the chalcogenide base, the ions nucleate on the chalcogen-rich regions within the base glass, resulting in a ternary that takes the form of a dispersed nanoscale metal ion-rich phase in a continuous glassy matrix [5]. This allows the electrolyte to have a relatively high resistivity (necessary for a high off resistance state), while containing large quantities of highly 


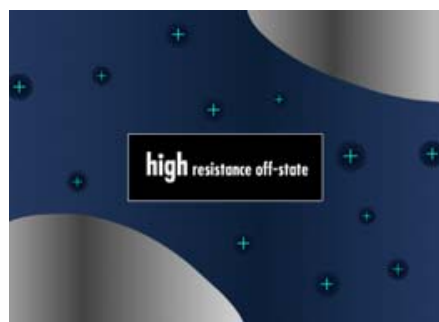

(a)

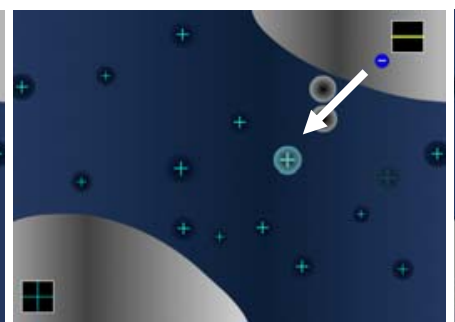

(b)

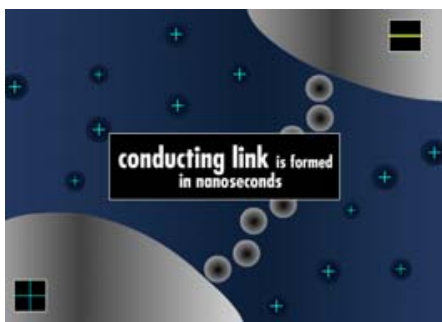

(c)

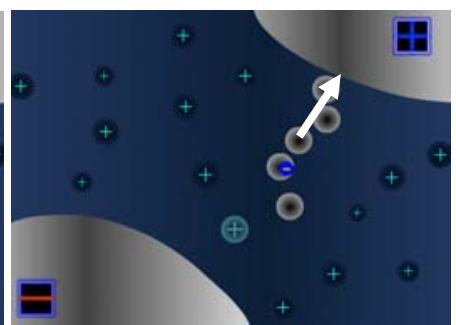

(d)

Fig. 1. Device operation for a coplanar series switch. (a) switch is off, (b) small bias forces electrons in cathode to reduce silver ions in substrate, (c) process halts when path is formed, and (d) process is reversed with application of reverse bias.

For an ion current to flow in an electrolyte, an oxidizable electrode is made positive (anode) with respect to an opposing electrode (cathode) and sufficient bias is applied, typically on the order of a few tenths of a volt or more. For $\mathrm{Ag}^{+}$-saturated $\mathrm{GeSe}_{2}$, silver at the anode is oxidized to form an excess of $\mathrm{Ag}^{+}$ions within the chalcogenide base glass. The applied field causes the ions to flow toward the cathode through the coordinated hopping mechanism described above. At the cathode, a reduction reaction occurs with injected electrons to re-form silver metal, as shown in Fig. 1(b). The number of atoms electrodeposited by the reduction of ions will correspond to the number of electrons that take part in the process (supplied by the external circuit). Each metal ion undergoing reduction will be balanced by a metal atom becoming oxidized to avoid the formation of an internal electric field due to the build up of charge. The process will continue until the programmed voltage/current limits supplied by the external power source is met. If the current limit is made sufficiently high $(\sim \mu \mathrm{A})$, a conductive silver bridge is formed which connects the two electrodes (Fig. 1(c)). Once this conduction path is formed, no further power is required to maintain it. To reverse this process, the electrodeposit is made positive with respect to the original oxidizable electrode, causing the dissolution of the metal bridge (Fig. 1(d)). During the dissolution of the electrodeposit, the balance is maintained by deposition of metal back onto the silver electrode. Once the electrodeposit has been completely dissolved, the process self-terminates. Note that for this process to occur, a metal ion-rich anode is required to induce appreciable ion current flow. Further, to be reversible, the opposing electrode must be made electrochemically inert (not oxidizable).

\section{Microwave Switch Design AND Operation}

The preliminary design of a microwave series switch exploiting this nanoionic behavior consisted of a simple twoelectrode coplanar geometry. A representative circuit layout can be seen in the microphotograph of Fig. 2. Two electrodes of dissimilar metals ( $\mathrm{Ag}$ anode, $\mathrm{Ni}$ cathode) are Au-plated to $1.5 \mu \mathrm{m}$ thick onto a $500 \mu \mathrm{m}$ thick quartz substrate $\left(\varepsilon_{\mathrm{r}}=3.9\right)$.
A gap of $10 \mu \mathrm{m}$ separates the two electrodes. Within this gap, a thin film $(\sim 100 \mathrm{~nm})$ of silver-saturated $\mathrm{GeSe}_{2}$ glass is deposited which represents the active area of the device. Several iterations provided the framework for an optimal geometry based on the nanoionic material characteristics and coplanar layout. The end result consisted of a $50-\Omega$ transmission line which tapers to a higher impedance (narrowed width) towards the gap. As will be discussed later, it is this tapering effect which helps to reduce capacitive coupling in the switch in the OFF state, but remains the primary source of loss in the ON state. A simple photoresist passivation layer atop the "active" area was implemented to provide protection.

To operate the device, a voltage of nominally $1 \mathrm{~V}$ and a current limit of $10 \mathrm{~mA}$ were used. Higher voltages induced faster growth rates, whereas higher current limits reduced the overall resistive loss of the electrochemically grown metal, but results in higher power requirements to operate the switch. The application of a positive voltage relative to the $\mathrm{Ni}$ (inert) electrode induces silver growth and enables the device to be turned ON. Reversing polarity of the applied voltage removes the electrochemically grown silver and forces the device into the OFF state. Fig. 3 is a microphotograph of the conductive metallic pathways that form when the device is ON. From the inset of Fig. 3, an atomic force microscope image shows that much of the silver growth occurs on the surface of the thin film.

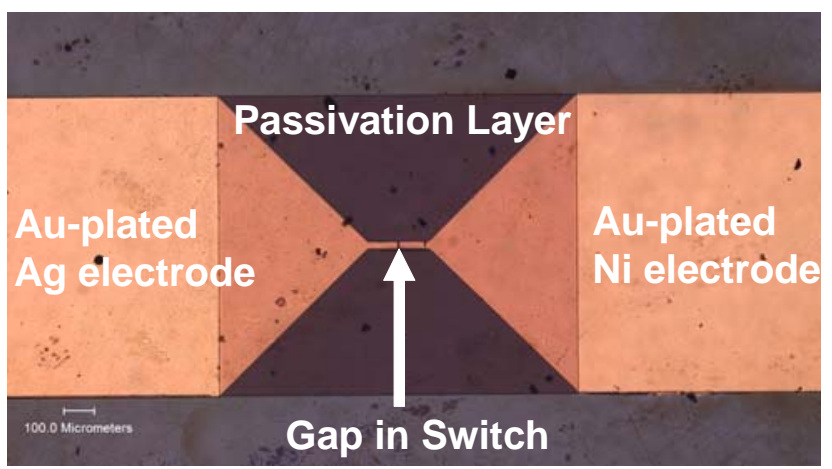


Fig. 2. Microphotograph of coplanar nanoionic switch design.

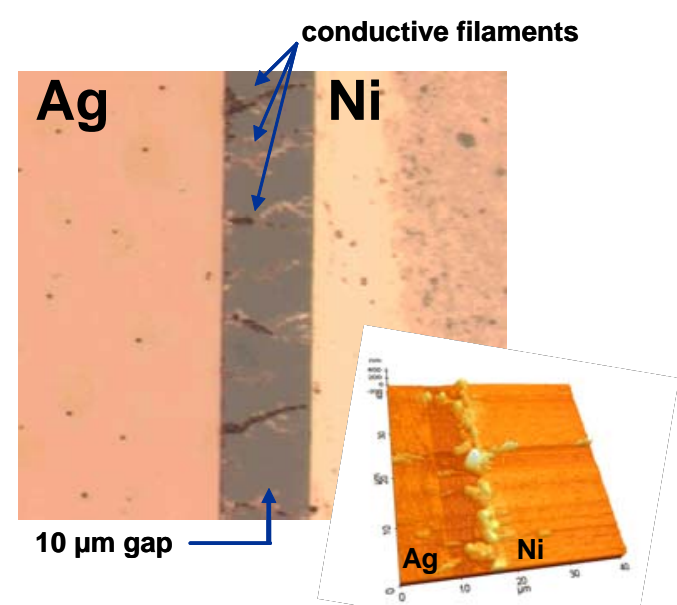

Fig. 3. Microphotograph (larger image in figure) and associated Atomic Force Microscope (AFM) image (smaller inset on right) of nanoionics-based switch in the ON state.

\section{MEASUREMENTS AND RESULTS}

\section{A. Insertion Loss and Isolation}

To measure the microwave performance of the device, test samples were individually diced and mounted onto a brass mounting fixture. The circuit was connected to an Agilent E8361A Vector Network Analyzer in order to measure ON state insertion loss and OFF state isolation. An Agilent E3646A DC Power Supply was utilized to provide the necessary voltage/current to change the state of the device (nominally $1 \mathrm{~V} / 10 \mathrm{~mA}(\mathrm{ON})$ and $-1 \mathrm{~V} / 10 \mathrm{~mA}(\mathrm{OFF})$ ). A plot of these results is shown in Fig. 4 for a typical nanoionic switch.

\section{Typical ON/OFF Characteristics of Nanoionics-based Switch}

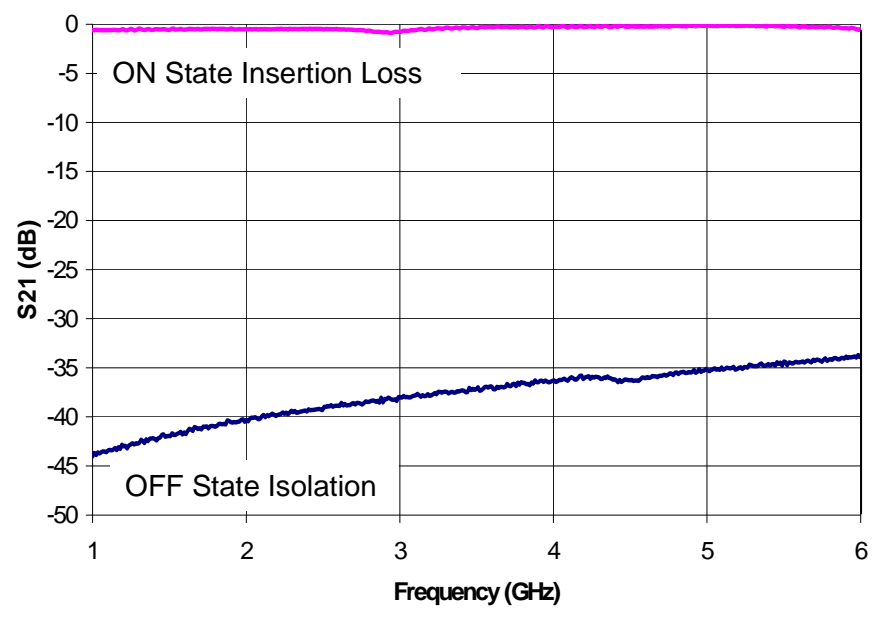

Fig. 4. The insertion loss (S21) of the switch in the ON state is better than $-0.5 \mathrm{~dB}$ over the 1 to $6 \mathrm{GHz}$ range (commercial ISM Band), while the isolation in the OFF state is better than $-35 \mathrm{~dB}$.

From the plot, we observe that an $\mathrm{ON}$ state insertion loss of $\sim 0.5 \mathrm{~dB}$ is realizable, with an OFF state isolation of better than $35 \mathrm{~dB}$ over the $\mathrm{DC}$ to $6 \mathrm{GHz}$ band. These measured results are comparable to MEMS and solid state-based RF switch performance in the same frequency range. At frequencies much greater than this, the series switch design tends to perform poorly, maintaining good insertion loss characteristics, but reducing isolation (due to capacitive edge coupling at the gap). Further, repeatability of this performance over several cycles also requires improvement.

\section{B. Linearity and Power Handling}

Power measurements were performed using an Anritsu ML2437A power meter and Anritsu MG3691B signal generator. A switch was mounted on the same brass mounting fixture while various frequencies $(500 \mathrm{MHz}-4 \mathrm{GHz})$ of varying power were fed into a switch. Attenuation pads at the input and output to the switch were used to reduce signal reflection within the system. Frequency and power range limits were determined by the dynamic range of the signal generator and available components.

From Fig. 5, we observe that devices typically demonstrated linearity over the range of measured power from $-20 \mathrm{dBm}$ to $+20 \mathrm{dBm}$, with device breakdown typically occurring at $\sim 400$ $500 \mathrm{~mW}$. Further, no change is evident in the power transfer curve at different frequencies of operation, demonstrating the device's wide bandwidth operation potential.

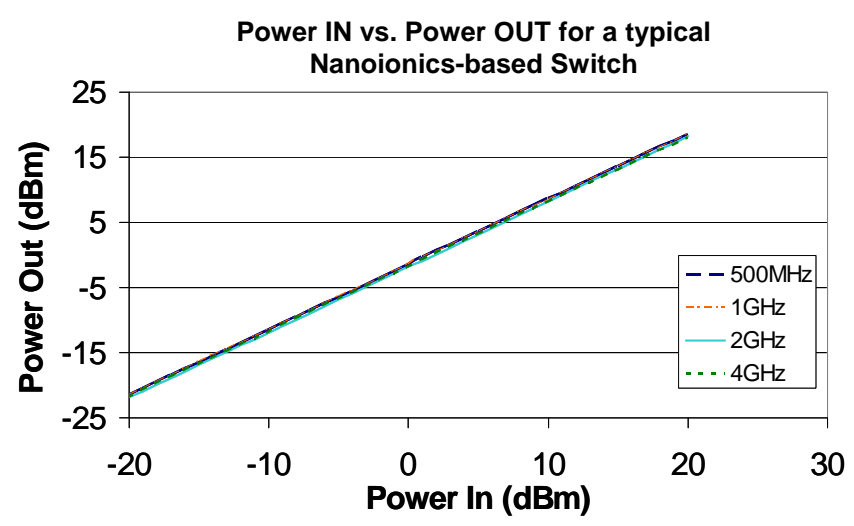

Fig. 5. Power transfer curve of a typical nanoionics-based switch over its operating range for frequencies between $500 \mathrm{MHz}$ and 4 $\mathrm{GHz}$.

\section{Speed}

The speed of operation of the nanoionic switch is a function of the distance the electrodeposit has to traverse. That is, the wider the gap between electrodes, the longer the response time of the switch. Although the switching speed was not 
directly measured, results inferred from [6] indicate that the electrodeposition rate of silver within a chalcogenide glass occurs at a velocity of approximately $1 \mathrm{~nm} / \mathrm{ns}$. Therefore, for a $10 \mu \mathrm{m}$ gap, a switching speed of around $10 \mu \mathrm{s}$ is expected. Obviously the smaller the gap size, the faster the switch operation, but other considerations such as OFF state isolation limit the optimization of speed, at least for a coplanar-type structure.

\section{Novel Microwave ApPlications}

The potential for novel device structures given this type of switch represents an attractive area which warrants further development. One such concept is that of a single-pole- $\mathrm{N}$ throw switch (SPNT). Since the active switching element is comprised of simply a thin film area, the addition of extra electrodes (ports) in contact with the active area makes possible the creation of SPNT switches, as shown in the diagram of Fig. 6. By merely applying the necessary voltage across the proper electrodes, a conductive pathway can be formed/dissolved amongst one of several different paths, limited only by available space and maximum coupling level requirements. Further, the ability to deposit vias of this nanoionic material allows for the formation of multilayer control circuits. This has the advantage of compacting circuit footprints and reducing overall circuit losses, while maintaining an unprecedented ease of integration.

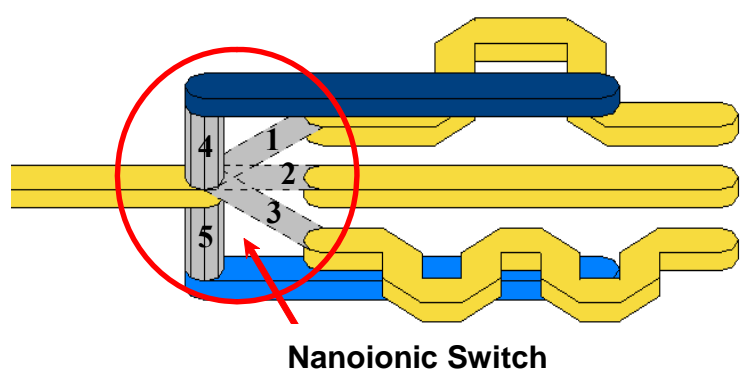

Fig 6. Drawing of a potential SP5T nanoionic switch, showing multiple horizontal, as well as vertical, switching paths.

One such application for this switch is in low loss phased array technology. For conventional discrete phase shifters based on a solid state or MEMS approach, approximately $2 \mathrm{~N}$ switches are required for an $N$-bit phase shifter, with 2-3 control lines per switch, contributing to the overall loss and complexity of the circuit. Implementing the nanoionic topology described above, we believe that it is possible to develop an $\mathrm{N}$-bit phase shifter with only 2 nanoionic switches and $N+2$ control lines. In doing so, line losses (and not switch loss) will be the primary contributor to total phase shifter loss. Further, circuit complexity will be minimized.

\section{Conclusion}

A novel microwave switch employing a nanoionics approach is discussed. The development and characterization of a coplanar series switch design indicates that, upon further optimization, this type of switch has the potential to perform comparably with the state-of-the-art solid state and MEMSbased microwave switches presently in development, with several inherent advantages, i.e., it is nonvolatile (fewer bias operations required), easily integrable, and makes possible the realization of novel device structures (e.g., multilayer circuit control, SPNT switches). However, further improvement of device performance (repeatability, losses, frequency range) is still necessary to compete with solid state and RF MEMS technology, but highly realizable.

\section{ACKNOWLEDGEMENT}

The authors wish to acknowledge the NASA Glenn Research Center Independent Research and Development Fund for support of this project.

\section{REFERENCES}

[1] M.N. Kozicki, C. Gopalan, M. Balakrishnan, M. Park, and M. Mitkova, "Non-volatile memory based on solid electrolytes," Proceedings of the 2004 Non-Volatile Memory Technology Symposium, 10-17, Orlando, Florida, November, 2004.

[2] M.N. Kozicki, M. Park, and M. Mitkova, "Nanoscale memory elements based on solid state electrolytes," IEEE Trans. Nanotechnology, vol. 4, no. 3, 331-338 (2005).

[3] S. Enderling, C.L. Brown, III, M. Balakrishnan, J. Hedley, J.T.M. Stevenson, S. Bond, C.C. Dunare, A.J. Harris, J.S. Burdess, M. Mitkova, M.N. Kozicki, A.J. Walton, "Integration of a novel electrochemical tuning scheme with MEMS surface micromachined resonators," $18^{\text {th }}$ IEEE International Conference on Micro Electro Mechanical Systems, 159-162 (2005).

[4] M.N. Kozicki, P. Maroufkhani, M. Mitkova, "Microfluidic control systems for biochips," BioDevice Interface Science and Technology Workshop, Scottsdale, AZ, September 2002

[5] M. Mitkova, M.N. Kozicki, "Silver incorporation in Ge-Se glasses used in programmable metallization cell devices," Journal of Non-Crystalline Solids, vol.299-302, part 2, 10231027 (2002)

[6] M.N. Kozicki, M. Yun, L. Hilt, A. Singh, "Application of programmable resistance changes in metal-doped chalcogenides," Proceedings of the 1999 Symposium on Solid State Ionic Devices, Electrochemical Society Proceedings Vol. 99-13, p. 298 (1999). 